\title{
Effect of antivenom therapy of Rhabdophis tigrinus (Yamakagashi snake) bites
}

Toru Hifumi ${ }^{*}$, Atsushi Sakai ${ }^{2}$, Akihiko Yamamoto ${ }^{3}$, Masahiro Murakawa ${ }^{4}$, Manabu Ato $^{5}$, Keigo Shibayama ${ }^{3}$, Hiroshi Kato ${ }^{6}$, Yuichi Koido ${ }^{6}$, Junichi Inoue ${ }^{7}, Y_{\text {Yko }} \mathrm{Abe}^{1}$, Kenya Kawakita ${ }^{1}$, Masanobu Hagiike ${ }^{1}$, Akihiko Ginnaga ${ }^{8}$ and Yasuhiro Kuroda ${ }^{1}$

\begin{abstract}
Background: Rhabdophis tigrinus (Yamakagashi snake) is a rear-fanged colubrid snake present throughout Russia and Asia. Its venom induces life-threatening hemorrhagic symptoms and severe disseminated intravascular coagulation with a fibrinolytic phenotype.

R. tigrinus antivenom manufactured by the immunization of horses to neutralize the venom has the risk of adverse events such as anaphylaxis and serum sickness disease. It should be used when benefit is greater than the risk of adverse effects; however, its efficacy has not been well evaluated.

Although our previous survey of nine cases demonstrated that seven of all cases treated with antivenom survived, the clinical characteristics and prognosis without antivenom administration remained unclear. We assumed that $R$. tigrinus antivenom administration overlaps self-recovery with supportive care. We aimed to determine the association between antivenom administration and outcome with further analyzed cases.
\end{abstract}

Methods: We retrospectively reviewed the records of the Japan Snake Institute between January 1, 1973 and December 31, 2013. Antivenom and without antivenom groups were compared with regard to baseline demographic features, treatment-related factors, and outcomes.

Results: In total, 34 patients were analyzed (97\% male, median age 37.5 years). Twenty-five patients were further examined from our previous study. On admission, the median levels of fibrinogen and fibrinogen degradation products were $35 \mathrm{mg} / \mathrm{dL}$ and $200 \mu \mathrm{g} / \mathrm{mL}$, respectively, and platelet counts were 107,000/mm ${ }^{3}$. The median disseminated intravascular coagulation score (defined by the Japanese Association of Acute Medicine) was 5. Antivenom was administered to 19 patients, with a median interval of $32 \mathrm{~h}$ between bite and antivenom administration. The in-hospital mortality rate was $12 \%$. In univariate analysis, baseline characteristics and laboratory data were not significantly different between the antivenom and without antivenom groups. Hospital mortality in the antivenom group was significantly better than that in the without antivenom group ( $0 \% \mathrm{vs} .26 .7 \%, P=0.03$ ). Moreover, the number of patients developing renal failure requiring hemodialysis was significantly lower in the antivenom group (5.3\% vs. $40.0 \%, P=0.03)$.

Conclusions: In our small retrospective study, antivenom administration was likely to be effective in the management of $R$. tigrinus bites. Apparently, further research is required to confirm its efficacy.

Keywords: Yamakagashi, Rhabdophis tigrinus, Antivenom

\footnotetext{
* Correspondence: hifumitoru@gmail.com

${ }^{1}$ Emergency Medical Center, Kagawa University Hospital, 1750-1 Ikenobe, Miki Kita, Kagawa 761-0793, Japan

Full list of author information is available at the end of the article
} 


\section{Background}

Rhabdophis tigrinus (Yamakagashi snake) is a rear-fanged venomous snake present throughout Russia and Asia [1]. Its venom induces life-threatening hemorrhagic symptoms and severe disseminated intravascular coagulation (DIC) with a fibrinolytic phenotype [2].

$R$. tigrinus antivenom manufactured by the immunization of horses to neutralize the venom has the risk of adverse events such as anaphylaxis and serum sickness disease $[1,2]$. It should be used when benefit is greater than the risk of adverse effects; however, its efficacy has not been well evaluated. Although our previous survey of nine cases demonstrated that seven of all cases treated with antivenom survived, the clinical characteristics and prognosis without antivenom administration remained unclear [3]. Further, theoretically, $R$. tigrinus antivenom only neutralizes the unbound venom and cannot restore organ function. Antivenom was administered after patients developed severe DIC in the study (the median interval between bite and antivenom administration was $35 \mathrm{~h}$ ) [2]. We assume that the $R$. tigrinus antivenom administration overlaps self-recovery with supportive care.

The present study therefore aimed to determine the association between antivenom administration and outcome with further analyzed cases.

\section{Methods}

The institutional review board of the Japan Snake Institute approved the present study.

\section{Patients and setting}

The Japan Snake Institute was established in 1968 to research medical application of snakes.

In clinical practice, physicians managing patients with snake bites usually ask for the assistance of the Japan Snake Institute, where diagnosis is confirmed according to laboratory data and clinical symptoms. Clinical data was routinely collected, and all cases of $R$. tigrinus bites were recorded in this institute. The records of the Japan Snake Institute were retrospectively investigated between January 1, 1973 and December 31, 2013.

\section{Diagnosis of $R$. tigrinus bites}

$R$. tigrinus bites were diagnosed based on the detailed information of snakes that patients observed and hemorrhagic symptoms including severe hypofibrinogenemia, and final diagnosis was recorded in a file of the Japan Snake Institute.

We also applied DIC diagnostic criteria for critically ill patients, as outlined by the Japanese Association of Acute Medicine (JAAM criteria) [4]; DIC was defined as a total score of $\geq 4$.

\section{Treatment of $R$. tigrinus bites}

The antivenom used against $R$. tigrinus bites was experimentally manufactured [1]. Severe adverse effects exclusively refer to anaphylactic shock in which the patient is at a risk of death because of antivenom administration.

\section{Data collection}

The following parameters were recorded: age, gender, date of injury, clinical symptoms, laboratory data, and DIC score as well as treatment-related factors and the outcomes including hospital mortality and renal failure requiring hemodialysis.

\section{Outcome measures}

The primary endpoint of the present study was to determine the association between antivenom administration and hospital mortality. The secondary outcome was to determine the association between antivenom administration and renal failure requiring hemodialysis after the acute phase of injury.

\section{Primary data analysis}

Statistical analysis was performed using JMP version 11 (SAS, Cary, NC, USA). Patient characteristics, treatmentrelated factors, and outcomes were compared between the antivenom group and the without antivenom group using Mann-Whitney $U$ test and $\chi^{2}$ test or, where appropriate, the Fisher exact test for categorical variables. $P$ values of $\leq 0.05$ alpha were considered statistically significant.

\section{Results}

Demographic data and clinical characteristics of all study patients

Over the 43-year study period, 34 patients were identified; the patient characteristics are summarized in Table 1 . We further analyzed 25 cases from the previous study [3]. All patients, except for one, were male, with a median age of 37.5 years. On admission, the median levels of fibrinogen and fibrinogen degradation products (FDPs) were $35 \mathrm{mg} / \mathrm{dL}$ and $200 \mu \mathrm{g} / \mathrm{mL}$, respectively, and platelet counts were $107,000 / \mathrm{mm}^{3}$. The mean DIC score was 5 .

Antivenom was administered to 19 patients, and the median interval between bite and antivenom administration was $32 \mathrm{~h}$. No apparent adverse effects were observed. DIC was treated with heparin in 14 patients. Seven patients developed renal failure requiring hemodialysis after the acute phase of the injury, and the in-hospital mortality rate for all the patients was $11.8 \%$.

\section{Comparison of clinical characteristics between the antivenom and without antivenom groups}

The comparison of clinical characteristics between the antivenom and without antivenom groups is summarized in Table 2. Baseline characteristics and laboratory 
Table 1 Population characteristics, $n=34$

\begin{tabular}{|c|c|}
\hline Population characteristics & Values \\
\hline Age (years) & $37.5(43.8)$ \\
\hline Gender, male, $n$ (\%) & $33(97.1)$ \\
\hline \multicolumn{2}{|l|}{ Date of getting injury (year) } \\
\hline 1973-1999 & $25(73.5)$ \\
\hline $2000-2013$ & $9(26.5)$ \\
\hline \multicolumn{2}{|l|}{ Clinical symptoms } \\
\hline Nasal bleeding, $n$ (\%) & $4(11.8)$ \\
\hline Gum bleeding, $n$ (\%) & $15(44.1)$ \\
\hline Bleeding from the bite sites, $n(\%)$ & $27(79.4)$ \\
\hline Headache, $n(\%)$ & $6(17.6)$ \\
\hline \multicolumn{2}{|l|}{ Laboratory data } \\
\hline Platelet counts $\left(\times 10^{4} / \mathrm{mm}^{3}\right)$ & $10.7(10.4)$ \\
\hline Fibrinogen (mg/dL) & $35(30)$ \\
\hline PT-INR & $5(4.38)$ \\
\hline $\mathrm{FDP}(\mu \mathrm{g} / \mathrm{mL})$ & $200(180)$ \\
\hline DIC score & $5(3)$ \\
\hline \multicolumn{2}{|l|}{ Treatment } \\
\hline Heparin, n (\%) & $14(41.2)$ \\
\hline FFP, n (\%) & $8(25.0)$ \\
\hline$P E, n(\%)$ & $4(11.8)$ \\
\hline Antivenom, n (\%) & $19(55.9)$ \\
\hline $\begin{array}{l}\text { Time interval between getting Yamakagashi } \\
\text { bites and antivenom administration (h) }\end{array}$ & $32(31)$ \\
\hline Severe adverse effects related to antivenom & $0(0)$ \\
\hline \multicolumn{2}{|l|}{ Outcome } \\
\hline Mortality, n (\%) & $4(11.8)$ \\
\hline Hospital stay & $9.5(9.5)$ \\
\hline Renal failure requiring hemodialysis, $n$ (\%) & $7(20.6)$ \\
\hline
\end{tabular}

Data are presented as median (interquartile, IQR) for continuous variables and $n$ (percentage) for categorical variables. PT-INR prothrombin time international ratio, FDP fibrinogen degradation products, DIC disseminated intravascular coagulation, FFP fresh frozen plasma, $P E$ plasma exchange, $S D$ standard deviation.

data were not significantly different between the two groups.

Heparin use in the antivenom group was significantly lower than that in the without antivenom group $(21.1 \%$ vs. $66.7 \%, P=0.01)$.

\section{Correlations between antivenom administration and outcomes}

Hospital mortality in the antivenom group was significantly better than that in the without antivenom group ( $0 \%$ vs. $26.7 \%, P=0.03$ ) (Figure 1 ). Moreover, the number of patients developing renal failure requiring hemodialysis was significantly lower in the antivenom group $(5.3 \%$ vs. $40.0 \%, P=0.03$ ) (Figure 2).
Table 2 Comparison between the antivenom and the without antivenom groups

\begin{tabular}{|c|c|c|c|}
\hline Characteristics & $\begin{array}{l}\text { Antivenom } \\
\text { group }(n=19)\end{array}$ & $\begin{array}{l}\text { Without antivenom } \\
\text { group }(n=15)\end{array}$ & $P$ value \\
\hline Age (years) & $37(40)$ & $43(50)$ & 0.93 \\
\hline Gender, male, $n(\%)$ & $18(94.7)$ & $15(100)$ & 1.00 \\
\hline $\begin{array}{l}\text { Date of injury, year } \\
(2000-2013), n(\%)\end{array}$ & $7(36.8)$ & $2(13.3)$ & 0.24 \\
\hline \multicolumn{4}{|l|}{ Clinical symptoms } \\
\hline Nasal bleeding, n (\%) & $1(5.3)$ & $3(20.0)$ & 0.07 \\
\hline Gum bleeding, n (\%) & $8(42.1)$ & $7(46.7)$ & 1.00 \\
\hline $\begin{array}{l}\text { Bleeding from the } \\
\text { bite sites, } n(\%)\end{array}$ & $16(84.2)$ & $11(73.3)$ & 0.67 \\
\hline Headache, $n$ (\%) & $3(15.8)$ & $3(20.0)$ & 1.00 \\
\hline \multicolumn{4}{|l|}{ Laboratory data } \\
\hline $\begin{array}{l}\text { Platelet counts } \\
\left(\times 10^{4} / \mathrm{mm}^{3}\right)\end{array}$ & $12.5(10.1)$ & 7.9 (11.6) & 0.21 \\
\hline Fibrinogen (mg/dL) & $42.5(20)$ & $31(43)$ & 0.34 \\
\hline PT-INR & $5.84(4.24)$ & $2.81(4.11)$ & 0.1 \\
\hline $\mathrm{FDP}(\mu \mathrm{g} / \mathrm{mL})$ & $236(185)$ & $160(214)$ & 0.06 \\
\hline DIC score & $5(4)$ & $4.5(3)$ & 0.6 \\
\hline \multicolumn{4}{|l|}{ Treatment } \\
\hline Heparin, $n$ (\%) & $4(21.1)$ & $10(66.7)$ & 0.01 \\
\hline$F F P, n(\%)$ & $3(15.8)$ & $5(38.5)$ & 0.22 \\
\hline PE & $1(5.3)$ & $3(20.0)$ & 0.30 \\
\hline
\end{tabular}

Data are presented as median (interquartile, IQR) for continuous variables and $n$ (percentage) for categorical variables. PT-INR prothrombin time international ratio, FDP fibrinogen degradation products, DIC disseminated intravascular coagulation, FFP fresh frozen plasma, PE plasma exchange, SD standard deviation.

\section{Discussion}

In the present study, we demonstrated that hospital mortality and the number of renal failure requiring hemodialysis following the acute phase of $R$. tigrinus bites were significantly better in patients receiving antivenom than in those not receiving antivenom. Previously, we demonstrated that the pathophysiology of $R$. tigrinus bites involves DIC with the fibrinolytic phenotype [3]. However, it seems that this DIC with fibrinolysis phenomenon does not persist throughout hospitalization and may be limited to the acute injury phase. The present survey revealed that in the acute phase, patients developed DIC with the fibrinolytic phenotype; however, $40 \%$ of patients without antivenom developed renal failure requiring hemodialysis in the later phase of the injury. Renal pathology has revealed that glomerular fibrin thrombi and tubular necrosis are responsible for renal failure associated with $R$. tigrinus bites [5]. Indeed, Gando et al. reported that 24 to $48 \mathrm{~h}$ after severe traumatic injury, DIC with the fibrinolytic phenotype changes to DIC with the thrombotic phenotype, which can result in the fatal multiple organ dysfunction syndromes (MODS) [6,7]. 


\begin{tabular}{cccc}
\hline & Dead & Survival & Total \\
\hline Antivenom group & 0 & 19 & 19 \\
Without-antivenom group & 4 & 11 & 15 \\
Total & 4 & 30 & 34 \\
\hline
\end{tabular}

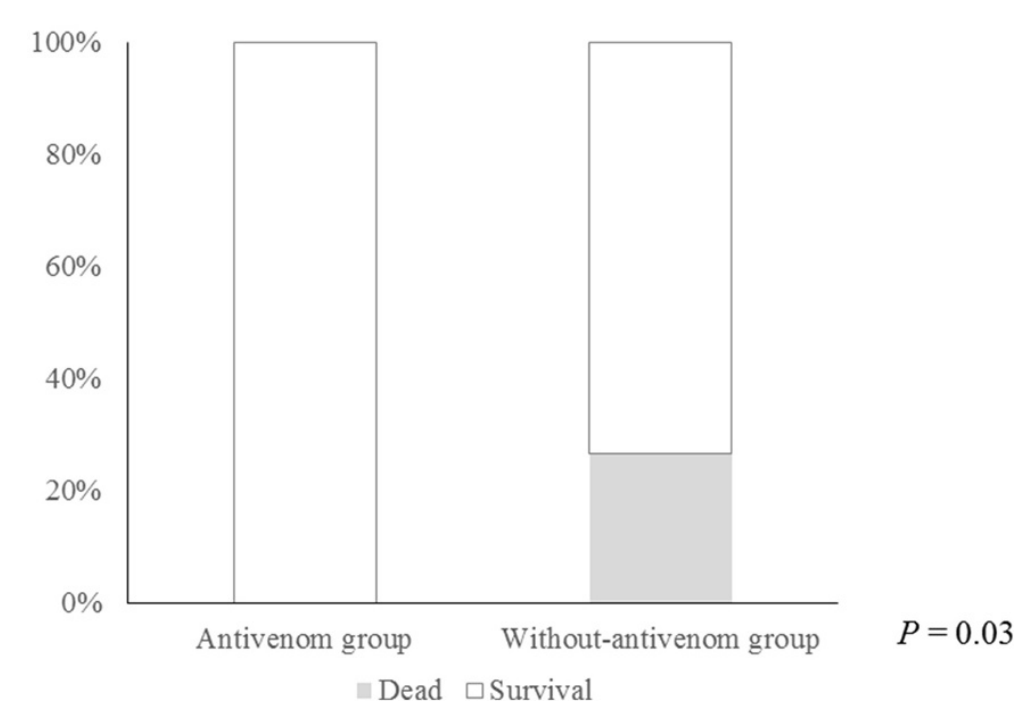

Figure 1 Comparison of hospital mortality between the antivenom and the without-antivenom groups. Hospital mortality in the antivenom group was significantly better than that in the without antivenom group ( $0 \%$ vs. $26.7 \%, P=0.03)$.

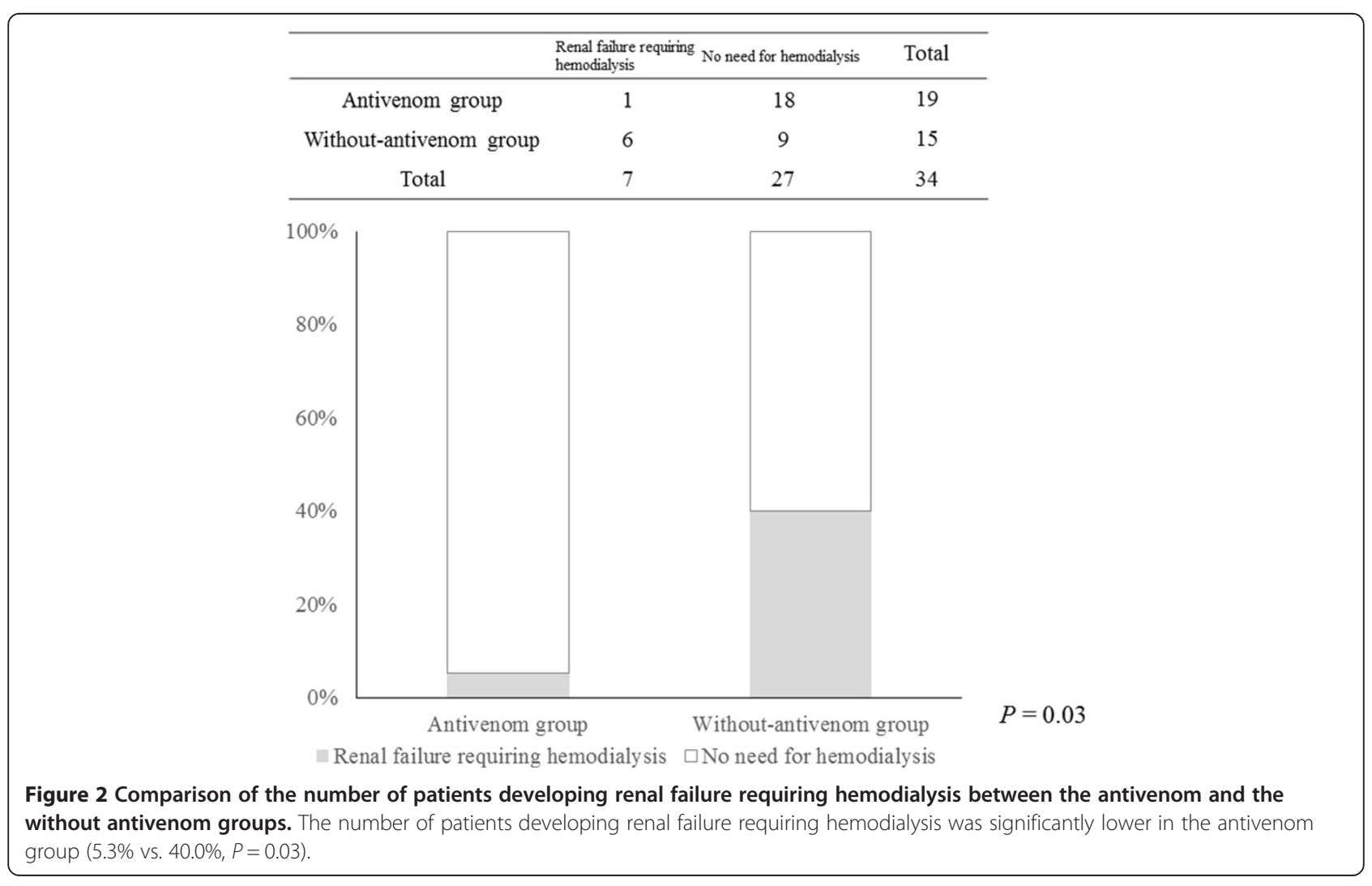


Gando et al. argued that the guiding principal in the treatment of DIC is the specific and vigorous treatment of the underlying disorder [6]. Considering our current understanding of the pathophysiology of $R$. tigrinus bites, it is obvious that managing DIC with heparin is contraindicated in the acute phase because patients develop bleeding manifestations [8]. On the other hand, antivenom represents a specific, definitive, and effective treatment in this phase. It appeared that administering $R$. tigrinus antivenom following bites can lead to complete clinical recovery without progression to MODS, even in the presence of severe DIC. Thus, antivenom effectively treats the acute symptoms and can prevent disease progression. If there is appropriate preparedness for anaphylaxis, antivenom should be used in patients with $R$. tigrinus bites.

A major adverse effect of antivenom is serum sickness disease, which usually occurred in 4-10 days after administration of antivenom [9]. Rashes, itching, joint pain, fever, lymphadenopathy, malaise, and renal failure are typical symptoms $[9,10]$. Because the number of patients developing renal failure requiring hemodialysis was significantly lower in the antivenom group, the close association between antivenom administration and renal failure was not considered. In the present study, although the numbers in the present survey are still too low to make any comprehensive assessment, the initial anaphylactic reaction rate was also lower than the $2.4 \%-9 \%$ rate observed with G. blomhoffii antivenom [11,12].

There are many limitations to the present study. Notably, the present study had a retrospective design and a relatively small sample size. Selection bias may also have been an issue because only cases reported to our center were used, and many cases may have remained undiagnosed or misdiagnosed because of the unfamiliar symptoms presented by this rare snakebite. Finally, because tissue plasminogen activator (t-PA) was not evaluated, the primary activation of fibrinogenolysis remains unclear. Furthermore, plasminogen activator inhibitor-1 (PAI-1), which induces the suppression of fibrinolysis, was not evaluated. Further study is required to clarify the pathophysiology of $R$. tigrinus bites.

\section{Conclusions}

In our small retrospective study, antivenom administration was likely to be effective in the management of $R$. tigrinus bites. Apparently, further research is required to confirm its efficacy.

\section{Competing interests}

The authors declare that they have no competing interests.

\section{Authors' contributions}

TH, AS, AY, YA, and YK collected the patient data. TH, MM, AG, HK, YK, Jl, YA, $\mathrm{KK}$, and $\mathrm{MH}$ treated patients. TH wrote the manuscript. MA, KS, and $Y K$ revised and edited the manuscript. All authors read and approved the final manuscript.

\section{Acknowledgements}

This study was supported by the Health Science Grants (2013-2015) from the Ministry of Health, Labour and Welfare of Japan.

\section{Author details}

'Emergency Medical Center, Kagawa University Hospital, 1750-1 Ikenobe, Miki Kita, Kagawa 761-0793, Japan. ${ }^{2}$ The Japan Snake Institute, Yabutsuka 3318, Ota, Gumma 379-2301, Japan. ${ }^{3}$ Department of Bacteriology II, National Institute of Infectious Disease, Gakuen 4-7-1, Musashimurayama-shi, Tokyo 208-0011, Japan. ${ }^{4}$ Department of Internal Medicine, Kaizuka Hospital, Hakosaki 7-7-27, Higashi-ku, Fukuoka 812-0053, Japan. ${ }^{5}$ Department of Immunology, National Institute of Infectious Disease, Toyama 1-23-1, Shinjuku-ku, Tokyo 162-8640, Japan. ${ }^{6}$ Division of Critical Care Medicine and Trauma, National Hospital Organization Disaster Medical Center, 3256 Midori-cho, Tachikawa, Tokyo 190-0014, Japan. ${ }^{7}$ Division of Critical Care Medicine and Trauma, Yamanashi Prefectural Central Hospital, 1-1-1 Fujimicho, Kofu, Yamanashi 400-8506, Japan. ${ }^{8}$ The Chemo-Sero-Therapeutic Research Institute (KAKETSUKEN), 1-6-1 Okubo, Kita-ku, Kumamoto-shi, Kumamoto 860-8568, Japan.

Received: 23 May 2014 Accepted: 23 June 2014

Published: 31 July 2014

\section{References}

1. Morokuma K, Kobori N, Fukuda T, Uchida T, Sakai A, Toriba M, Ohkuma K, Nakai K, Kurata T, Takahashi M: Experimental manufacture of equine antivenom against yamakagashi (Rhabdophis tigrinus). Jpn J Infect Dis 2011, 64:397-402.

2. Dart RC, McNally J: Efficacy, safety, and use of snake antivenoms in the United States. Ann Emer Med 2001, 37:181-188.

3. Hifumi T, Sakai A, Yamamoto A, Murakawa M, Ato M, Shibayama K, Ginnaga A, Kato H, Koido Y, Inoue J, Abe Y, Kawakita K, Hagiike M, Kuroda Y: Clinical characteristics of yamakagashi (Rhabdophis tigrinus) bites: a national survey in Japan, 2000-2013. J Intensive Care 2014, 2:19.

4. Gando S, Iba T, Eguchi Y, Ohtomo Y, Okamoto K, Koseki K, Mayumi T, Murata A, Ikeda T, Ishikura H, Ueyama M, Ogura H, Kushimoto S, Saitoh D, Endo S, Shimazaki S, Japanese Association for Acute Medicine Disseminated Intravascular Coagulation (JAAM DIC) Study Group: A multicenter, prospective validation of disseminated intravascular coagulation diagnostic criteria for critically ill patients: comparing current criteria. Crit Care Med 2006, 34:625-631.

5. Sakai T, Hatsuse M, Sawai Y: Study on the pathogenesis of envenomation by the Japanese Colubrid Snake, Yamakagashi, Rhabdophis tigrinus. Snake 1990, 22:11-19.

6. Gando S, Sawamura A, Hayakawa M: Trauma, shock, and disseminated intravascular coagulation: lessons from the classical literature. Ann Surg 2011, 254:10-19.

7. Gando S: Acute coagulopathy of trauma shock and coagulopathy of trauma: a rebuttal. You are now going down the wrong path. J Trauma 2009, 67:381-383.

8. Sakai A: Diagnosis and treatment of snakebite by Mamushi and Yamakagashi. Chudoku kenkyu 2013, 26:193-199.

9. Lundquist AL, Chari RS, Wood JH, Miller GG, Schaefer HM, Raiford DS, Wright KJ, Gorden DL: Serum sickness following rabbit antithymocyte-globulin induction in a liver transplant recipient: case report and literature review. Liver Transp/ 2007, 13:647-650.

10. Davies KA, Mathieson P, Winearls CG, Rees AJ, Walport MJ: Serum sickness and acute renal failure after streptokinase therapy for myocardial infarction. Clin Exp Immunol 1990, 80:83-88.

11. Hifumi T, Yamamoto A, Morokuma K, Okada I, Kiriu N, Ogasawara T, Hasegawa E, Kato H, Inoue J, Koido Y, Takahashi M: Clinical efficacy of antivenom and cepharanthine for the treatment of Mamushi (Gloydius blomhoffii) bites in tertiary care centers in Japan. Jpn J Infect Dis 2013, 66:26-31.

12. Hifumi T, Yamamoto A, Morokuma K, Ogasawara T, Kiriu N, Hasegawa E, Inoue J, Kato H, Koido Y, Takahashi M: Surveillance of the clinical use of mamushi (Gloydius blomhoffii) antivenom in tertiary care centers in Japan. Jpn J Infect Dis 2011, 64:373-376.

doi:10.1186/s40560-014-0044-5

Cite this article as: Hifumi et al.: Effect of antivenom therapy of Rhabdophis tigrinus (Yamakagashi snake) bites. Journal of Intensive Care 2014 2:44. 